\title{
Effect of Antioxidants on the Outcome of Therapy in Paraquat-intoxicated Patients
}

\author{
Nastaran Eizadi-Mood ${ }^{1}$, Ali Mohammad Sabzghabaee ${ }^{2^{*}}$, \\ Ahmad Yaraghi ${ }^{1}$, Kamran Montazeri ${ }^{1}$, Mojgan Golabi ${ }^{3}$, Alireza \\ Sharifian $^{3}$ and Shirinsadat Badri ${ }^{2}$ \\ ${ }^{1}$ Department of Anesthesiology and Intensive Care, School of Medicine, ${ }^{2}$ Isfahan Clinical Toxicology Research \\ Center, ${ }^{3}$ Noor General Teaching Hospital, Isfahan University of Medical Sciences, Isfahan, Iran.
}

\begin{abstract}
Purpose: The present study was conducted to determine the effect of vitamins $C$ and $E$ administration on the outcome of therapy in patients with paraquat intoxication admitted in the Poisoning Emergency Department (PED) of Noor Teaching General Hospital, Isfahan, Iran.

Methods: We studied retrospectively medical records of 186 patients admitted to the PED in two different study periods: 157 patients were evaluated from 1985 to 2001 (first study period) in which patients received conventional treatment protocol consisting of fluid replacement, oral absorbents, haemodialysis, etc, Also, 29 other patients were assessed from 2001 to 2005 (second study period) in which vitamins $C$ and $E$ had been administered to the patients, in addition to the conventional protocol. The patients' mortality rate after the first study period was compared to that of the second one, with regard to the impact of the additional therapeutic intervention (use of antioxidant vitamins).

Results: Most of the patients (40.5\%) had ingested more than $20 \mathrm{~mL}$ of $20 \%$ paraquat solution and were admitted to the hospital during the first $4 \mathrm{~h}$ after exposure. Paraquat poisoning was more common in men (76.6\%), young adults in the age group 19 - 34 (47\%) and during summer (43.3\%). Mean ( \pm $S E)$ length of hospital stay was $62.6 \pm 15.6 \mathrm{~h}$. Overall mortality rates were 31 and $55.2 \%$ in the first and second periods, respectively.

Conclusion: Although the addition of antioxidants (vitamins $C$ and E) to the conventional therapy did not reduce the mortality rate, other dependent variables including different doses of antioxidants may be considered for future studies.
\end{abstract}

Keywords: Paraquat poisoning, Vitamin C, Vitamin E, Outcome 


\section{INTRODUCTION}

Paraquat poisoning is the most common cause of fatal herbicide intoxication and most cases result from deliberate ingestion. The major cause of death in paraquat poisoning is respiratory failure due to an oxidative insult to the alveolar epithelium with subsequent obliterating fibrosis [1]. Currently, there are no effective pharmacological antagonists for paraquat and there are no chelating agents capable of binding the poison in blood or other tissues, and so the management of paraquat poisoning has remained mostly supportive and directed toward decreasing its absorption or enhancing its elimination [1].

Despite the best therapeutic efforts, paraquat presents a high mortality rate even when the patient ingests a minimal amount. Recognizing the fact that paraquat induces its toxic effects via oxidative stress-mediated mechanisms [2], further management should theoretically be directed towards reducing the acute alveolitis and pulmonary fibrosis by the use of antioxidants [2], as has been investigated in some studies. The two studies that evaluated the effect of melatonin as an antioxidant on the cell damage and outcome of paraquat-intoxicated rats found that melatonin increases survival time and provides beneficial effects against paraquat toxicity [3,4]. These effects were also demonstrated in another study with the antioxidant, $\mathrm{N}$-acetylcysteine [5].

Vitamins $\mathrm{C}$ and $\mathrm{E}$ are well-known antioxidants which rapidly react with radicals and interfere with lipid peroxidation. High concentrations of ascorbic acid are found naturally in the fluid of the lung to protect against free radicals generated by toxic chemicals in air. Accordingly, it has been assumed that both vitamins $\mathrm{C}$ and $\mathrm{E}$ are consumed during radical reactions [6,7].

In one study, after $48 \mathrm{~h}$ intraperitoneal administration of paraquat to rats, the vitamin $C$ concentration in the lungs was significantly decreased, indicating that vitamin $C$ directly reflected the oxidative stress in the lungs [8]. The other study [9] indicates that increasing total antioxidant status by high doses of vitamin $C$ could be useful as a free radical scavenger for paraquat-poisoned patients. Such protective effects have been attributed to the ability of vitamin $C$ to quench radicals generated by redox cycling of paraquat [9].

The role of vitamin $E$ in paraquat toxicity was demonstrated in several studies where deficiency of vitamin $E$ potentiated the development of acute paraquat toxicity in animals. In two different studies, vitamin E deficiency decreased survival and worsened histological lung damage in rats [10] and significantly reduced the LD50 in mice [11] exposed to paraquat. Furthermore, the potentiation of acute paraquat toxicity by vitamin $E$ deficiency was reversed by administration of vitamin $E$ [10]. The results of another study suggest that alphatocopherol (vitamin E) delivered directly to the lung in a liposomal formulation $24 \mathrm{~h}$ prior to paraquat administration, confers protection against paraquat-induced lung damage [12]. Although the mechanism(s) by which vitamin $E$ protects against paraquat toxicity is not understood, it may be attributed to its antioxidant properties in preventing lipid peroxidation or inhibiting the generation of superoxide anion and its toxicity [1].

Isfahan is the second city of Iran and is located in the central part of the country. Noor General Teaching Hospital is a referral university hospital of this region, especially for toxicologic emergencies. In this hospital over the years, the treatment protocol for the management of paraquat intoxication has undergone changes. Prior to 2001, treatment consisted of gastrointestinal decontamination using Fuller's Earth or Hewitt, activated charcoal, dexamethasone, $\mathrm{N}$-acetylcysteine (NAC) and haemodialysis. After 2001, vitamins $C$ and $E$ were added to this conventional protocol. Since almost all cases of paraquat intoxication are referred to this hospital, the objective of this study was to assess the effect of the addition of vitamin C 
and vitamin $\mathrm{E}$ to the earlier treatment protocol on the outcome of therapy in patients with paraquat intoxication.

\section{METHODS AND PATIENTS}

The present study was carried out retrospectively in the Poisoning Emergency Department of Noor General Teaching Hospital, Noor, Isfahan Province, Iran. Between 1985 and 2005, 186 adult patients with paraquat intoxication, as confirmed by urine dithionate testing, were evaluated. The exclusion criteria included patients who had renal or hepatic disorders at the time of hospitalization.

The basic demographic data of the patients as well as information about the season in which poisoning occurred, amount of paraquat ingested (as stated by the patient or or someone close to him/her); time from ingestion to admission, length of hospitalization; poisoning-associated complications such as renal, hepatic or pulmonary failure; treatment procedures (haemodialysis, drugs, antioxidants, etc); time of initiating haemodialysis after admission; dosage of vitamins $\mathrm{C}$ and $\mathrm{E}$; and patients' mortality rate, were collected in special forms from the patients' files in the records department of the hospital.

The medical records of 157 patients were evaluated from 1985 to 2001 (first study period) in which patients received conventional treatment protocol consisting of fluid replacement, oral absorbents, haemodialysis, etc. Twenty nine medical records of eligible patients who were admitted from 2001 to 2005 were assessed (second study period) in which various amounts of vitamins $C$ and $E$ had been administered to patients, in addition to the conventional protocol. During this period, the patients received daily $100-200 \mathrm{mg}$ of vitamin E intramuscularly and $2400-3600$ $\mathrm{mg}$ of vitamin $\mathrm{C}$ as intravenous infusion for 3 -5 days.

\section{Data analysis}

The patients' mortality rate in the first study period was compared to that of the second study period in order to determine the impact of the new therapeutic modalities (incorporating the antioxidant vitamins). All statistical analyses were performed by Spearman test using SPSS for Windows, version 13.0.

\section{RESULTS}

The basic demographic characteristics of the patients and the mortality rates in these two study periods are shown in Table 1. Paraquat poisoning was more common in men (76.6 $\%)$, young adults, i.e., age group $19-34$ years $(47 \%)$, and during summer $(43.3 \%)$. Most of the patients (40.5\%) had ingested more than $20 \mathrm{~mL}$ of $20 \%$ paraquat solution and were admitted to the hospital during the first $4 \mathrm{~h}$ following exposure, as stated by the patient or someone close to him/her. Haemodialysis was performed on patients mostly during 4 - $8 \mathrm{~h}$ after admission (63.2\%). Length of hospital stay (mean \pm SEM) was $62.6 \pm 15.6 \mathrm{~h}$.

Table 1: Frequency distribution (\%) of sex, age group and mortality rate in paraquat-poisoned patients for two study periods

\begin{tabular}{lccccccc} 
& Total & \multicolumn{2}{c}{ Sex } & \multicolumn{2}{c}{ Age group (years) } & \multirow{2}{*}{$\begin{array}{c}\text { Mortality } \\
\text { rate (\%) }\end{array}$} \\
\cline { 2 - 6 } Study period & & Male & Female & $<19$ & $\mathbf{1 9 - 3 4}$ & $>\mathbf{3 4}$ & \\
\hline First (1985-2001) & 157 & $108(69 \%)$ & $49(31 \%)$ & $54(34.5 \%)$ & $63(40 \%)$ & $40(25.5 \%)$ & $(31 \%)$ \\
Second (2001-2005) & 29 & $21(72.4 \%)$ & $8(27.6 \%)$ & $8(27.6 \%)$ & $14(48.3 \%)$ & $7(24.1 \%)$ & $(55.2 \%)$ \\
\hline
\end{tabular}


Renal failure concomitant with hepatic and pulmonary dysfunction was observed in $75 \%$ of cases. During the second period, paraquat poisoning in $55.2 \%$ of the patients resulted in death, with most of these occurring on the first day of admission. This contrasts with a mortality rate of $31 \%$ for patients in the first period of the study.

\section{DISCUSSION}

Acute paraquat poisoning is a major public health problem in many developing countries. Despite improvements in medical therapy, mortality rate for paraquat-poisoned patients remains tragically high. In our study, mortality rate was not reduced even after inclusion of antioxidant vitamin therapy. High mortality could be due to high amounts of paraquat ingested by the patients as most of the patients had ingested more than $20 \mathrm{~mL}$ of the poison. This high level of poison intake can cause multiple organ dysfunctions, leading to complications such as cardiogenic shock, acute pulmonary fibrosis, renal and hepatic failure, and ultimately, death in $24-48 \mathrm{~h}$ [2]. Most of the patients developed multiple organ dysfunctions.

The time interval between exposure and treatment initiation was also important. Although patients were referred to the hospital mostly during the first 4 hours following exposure, most of the patients died due to acute intoxication regardless of therapy. This could be attributed to the rapid absorption of this poison via the gastrointestinal tract.

Although, a few reports have shown the beneficial therapeutic effect of antioxidants in paraquat poisoning $[9,13,14]$, the present study indicate that in spite of the fact that vitamins $C$ and $E$ were administered for more than 2 days in most of the paraquatintoxication cases in order to prevent the pulmonary fibrosis, the mortality rate data showed that antioxidant therapy did not produce any beneficial outcome. None of the patients who ingested $>10 \mathrm{~mL}$ of paraquat survived. Thus, the main prognostic factor appears to be the quantity of paraquat ingested, as previous studies have indicated, including one report in 1979 which stated that at any given time after ingestion, the plasmaparaquat concentrations of patients who died usually exceeded those of survivors [15]. Exposure to lesser amount of paraquat, and lower degrees of renal, hepatic and pulmonary failure on admission, are good prognostic factors of survival after acute poisoning; consequently, antioxidant therapy is unlikely to improve the prognosis of paraquat intoxication.

The results of the present study are consistent with some previous studies. One related study conducted on guinea pigs indicated that alteration of endogenous ascorbic acid (vitamin C) levels did not alter biochemical response to paraquat [16]. However, the results of another study on rabbit kidney cortex slices did show that ascorbic acid is able to inhibit the accumulation of paraquat, and therefore may serve as an antidote for paraquat toxicity [17]. There have also been contradictory reports about the usefulness of vitamin $E$ in paraquat toxicity. While one study showed that vitamin $E$ inhibits the induction of cytotoxic effects of paraquat on cultured rat hepatocytes [18], others conclude that there is no protective influence of vitamin $E$ on paraquat intoxication [19-21].

\section{CONCLUSION}

Although adding antioxidants (vitamins $\mathrm{C}$ and E) to our conventional therapy did not reduce the mortality rate in patients who suffered paraquat poisoning, other variables such as inadequate dose of antioxidants, as well as variables associated with paraquat poisoning, also need to be considered. Thus, further well-designed prospective studies are needed to evaluate the outcome and mortality rate among the different treatment protocols. The failure of current treatments in the management of paraquat poisoning not only calls for large prospective clinical trials 
on the treatment of paraquat poisoning, but also for urgent preventive measures.

\section{ACKNOWLEDGEMENT}

This research was supported financially by the Vice-Chancellery of Research of Isfahan University of Medical Sciences, and designed and conducted by the Isfahan Clinical Toxicology Research Center (http://ctrc. mui.ac.ir). The authors would like to thank the academic faculty members of the Anesthesiology Department of Isfahan University of Medical Sciences as well as colleagues and staff of the Poisoning \& Emergency Department of Noor Teaching General Hospital for their valuable support.

\section{REFERENCES}

1. Suntres ZE. Role of antioxidants in paraquat toxicity. Toxicology 2002; 180: 65-77.

2. Haddad LM, Shannon MW, Winchester JF, Borron SW, Burns MJ. Haddad and Winchester's Clinical Management of Poisoning and Drug Overdose. Philadelphia: Elsevier Saunders, 2007; $p 1197$.

3. Gocgeldi E, Uysal B, Korkmaz A, Ogur R, Reiter RJ, Kurt B, Oter S, Topal T, Hasde $M$. Establishing the use of melatonin as an adjuvant therapeutic against paraquat-induced lung toxicity in rats. Exp Biol Med (Maywood). 2008; 233: 1133-1141.

4. Ramirez-Zambrano E, Zambrano E, Rojas G, Zambrano M, Teneud L. [Protective effect of melatonin and sodium thiosulphate on histopathology and ultrastructure of the kidney in rats with acute paraquat poisoning]. Invest Clin. 2007; 48: 81-89.

5. Yeh ST, Guo HR, Su YS, Lin HJ, Hou CC, Chen HM, Chang MC, Wang YJ. Protective effects of $\mathrm{N}$-acetylcysteine treatment post acute paraquat intoxication in rats and in human lung epithelial cells. Toxicology. 2006; 223: 181190.

6. Carr A, Frei B. Does vitamin $C$ act as a pro-oxidant under physiological conditions? FASEB J. 1999; 13:1007-1024.

7. Menzel DB. The toxicity of air pollution in experimental animals and humans: the role of oxidative stress. Toxicol Lett. 1994; 72: 269277.
8. Ikeda K, Kumagai $Y$, Nagano Y, Matsuzawa N, Kojo $S$. Change in the concentration of vitamins $C$ and $E$ in rat tissues by paraquat administration. Biosci Biotechnol Biochem. 2003; 67: 1130-1131.

9. Hong SY, Hwang KY, Lee EY, Eun SW, Cho SR, Han CS, Park YH, Chang SK. Effect of vitamin $C$ on plasma total antioxidant status in patients with paraquat intoxication. Toxicol Lett. 2002; 126: 51-59.

10. Block ER. Potentiation of acute paraquat toxicity by vitamin E deficiency. Lung. 1979; 156: 195203.

11. Bus JS, Aust SD, Gibson JE. Lipid peroxidation: a possible mechanism for paraquat toxicity. Res Commun Chem Pathol Pharmacol. 1975; 11: 31-38.

12. Suntres ZE, Hepworth SR, Shek PN. Protective effect of liposome-associated alpha-tocopherol against paraquat-induced acute lung toxicity. Biochem Pharmacol. 1992; 44: 1811-1818.

13. Cappelletti G, Maggioni MG, Maci R. Apoptosis in human lung epithelial cells: triggering by paraquat and modulation by antioxidants. Cell Biol Int. 1998; 22: 671-678.

14. Landaeta G, Fernandez $Y$, Sutil $R$, Aular $Y$, Marquez M, Reigosa A. Histological changes in lung of rats poisoned with Paraquat and treated with vitamin $E$ and Dexametasone. Salus. 2008; 12: 69-80.

15. Proudfoot AT, Stewart MS, Levitt T, Widdop $B$. Paraquat poisoning: significance of plasmaparaquat concentrations. Lancet. 1979; 2: 330332.

16. Sullivan TM, Montgomery MR. Ascorbic acid nutritional status does not affect the biochemical response to paraquat. Fund Appl Toxicol. 1984; 4: 754-759.

17. Fujimoto $Y$, Nakatani $E$, Horinouchi $M$, Okamoto $K$, Sakuma S, Fujita T. Inhibition of paraquat accumulation in rabbit kidney cortex slices by ascorbic acid. Res Commun Chem Pathol Pharmacol. 1989; 65: 245-248.

18. Watanabe N, Shiki Y, Morisaki N, Saito $Y$, Yoshida $S$. Cytotoxic effects of paraquat and inhibition of them by vitamin E. Biochim Biophys Acta. 1986; 883: 420-425.

19. Eckert $H$, Schnorr $R$, Renner $H$, Oddoy $A$, Lisotschkin BG. Studies of lung damage by paraquat. 2. Effect of vitamin $E$ on acute lung damage caused by paraquat. $Z$ Erkr Atmungsorgane. 1987; 169: 147-152.

20. Redetzki HM, Wood CD, Grafton WD. Vitamin E and paraquat poisoning. Vet Hum Toxicol. 1980; 22: 395-397.

21. Yasaka T, Okudaira K, Fujito H, Matsumoto J, Ohya I, Miyamoto Y. Further studies of lipid peroxidation in human paraquat poisoning. Arch Intern Med. 1986; 146: 681-685. 